\title{
Pulse Oximetry Markers for Cardiovascular Disease in Sleep Apnea
}

\author{
Margot Deviaene ${ }^{1}$, Pascal Borzée ${ }^{2}$, Bertien Buyse ${ }^{2}$, Dries Testelmans ${ }^{2}$, Sabine Van Huffel ${ }^{1}$, \\ Carolina Varon ${ }^{1}$ \\ ${ }^{1}$ KU Leuven, Department of Electrical Engineering (ESAT), STADIUS Center for Dynamical \\ Systems, Signal Processing and Data Analytics, Leuven, Belgium \\ ${ }^{2}$ UZ Leuven, Department of Pneumology, Leuven, Belgium
}

\begin{abstract}
Patients suffering from sleep apnea have an increased risk to develop cardiovascular diseases. Evidence suggests that the apnea hypopnea index (AHI) does not correlate well with this risk, therefore, there is a clinical need to define markers beyond the AHI to phenotype sleep apnea. This study investigates the use of pulse oximeter parameters to determine the cardiovascular status of sleep apnea patients. Oxygen saturation $\left(\mathrm{SpO}_{2}\right)$ and pulse photoplethysmography (PPG) features were extracted around $\mathrm{SpO} \mathrm{O}_{2}$ drops and averaged per patient. A random forest backwards wrapper was used to extract the feature set which could differentiate best between cardiovascular controls and patients who suffered from a cardiovascular event. A dataset of 975 patients was used for this study, 90 of them were used for training.

The results show that sleep apnea patients with a cardiovascular comorbidity tend to have more severe oxygen desaturations, and often do not have a complete resaturation to their baseline $\mathrm{SpO}_{2}$. A decreased variability in the $P P G$ pulse upslope, could point to less arousals in these patients. The classifier based on the $\mathrm{SpO}_{2}$ features combined with age obtained the best performance with an averaged test AUC of $77.7 \%$.
\end{abstract}

\section{Introduction}

Sleep apnea is the most common sleep related breathing disorder, causing repetitive cessations of breathing that lead to drops in the blood oxygen saturation $\left(\mathrm{SpO}_{2}\right)$ and/or to arousals [1]. These arousals allow the patient to start breathing again, but will also disturb their sleep, leading to daytime sleepiness and its associated decrease in quality of life. Moreover, untreated sleep apnea increases the risk to develop cardiovascular diseases [2]. This deterioration of the cardiovascular system has been associated with intrathoracic pressure changes, oxygen desaturations and arousals due to apneic events. However, the exact pathways of this association are not yet fully understood, but studies suggest that treatment of sleep apnea could decrease the cardiovascular risk [2]. Therefore, timely diagnosis and treatment of sleep apnea is of utmost importance. Diagnosis of sleep apnea is nowadays done using a full night in-hospital polysomnography (PSG). This is a timeconsuming, uncomfortable and expensive assessment that has as main outcome the apnea-hypopnea index (AHI), which is a count of the number of respiratory events per hour of sleep [1]. Clinically an AHI threshold of 15 is used for diagnosis.

This parameter, however, does not take into account the duration or type of respiratory events. Hence, it was shown that the AHI is not a good marker for cardiovascular comorbidities when adjusted for confounding factors such as age and BMI [3]. Moreover, the recent population-based HypnoLaus study measured an AHI larger than 15 in more than $50 \%$ of the included middle-aged men [4].

These studies show the need for an improved phenotyping of sleep apnea in order to achieve a better patient selection and treatment prioritization. In this study, the presence of cardiovascular comorbidities was chosen as patient differentiator. As such, the effect of sleep apnea on the cardiovascular system can be studied further. Moreover, the goal is to find markers in the non-obtrusive pulse oximetry signal in order to enable a wearable assessment, which could be brought to the home environment.

\section{Methods}

\subsection{Datasets}

For this study, three datasets collected at the sleep lab of the University Hospitals Leuven were used, containing PSG recordings together with a cardiovascular assessment of the patient at the time of the PSG. An overview of the demographics of the three datasets can be found in Table 1. Dataset 1 and 3 contain patients diagnosed with sleep apnea (AHI $\geq 15$ ), while the second dataset contains subjects only suspected to have sleep apnea. This led to a full range 
Table 1. Overview of some averaged patient demographics per dataset and for each cardiovascular comorbidity class (cardiac controls $\mathrm{C}$, symptoms $\mathrm{S}$, events $\mathrm{E}$ ).

\begin{tabular}{|l|cc|ccc|ccc|ccc|}
\hline \hline & \multicolumn{2}{|c|}{ Train set } & \multicolumn{3}{|c|}{ Dataset 1 } & \multicolumn{3}{c|}{ Dataset 2 } & \multicolumn{3}{c|}{ Dataset 3 } \\
& $\mathrm{C}$ & $\mathrm{E}$ & $\mathrm{C}$ & $\mathrm{S}$ & $\mathrm{E}$ & $\mathrm{C}$ & $\mathrm{S}$ & $\mathrm{E}$ & $\mathrm{C}$ & $\mathrm{S}$ & $\mathrm{E}$ \\
\hline \hline \# patients & 45 & 45 & 27 & 547 & 14 & 46 & 140 & 11 & 50 & 44 & 6 \\
\hline Age & 47.7 & 65.3 & 50.9 & 51.3 & 59.9 & 41.5 & 51.5 & 61.7 & 47.3 & 47.4 & 54.2 \\
(years) & \pm 11.8 & \pm 10.0 & \pm 7.9 & \pm 11.4 & \pm 7.4 & \pm 11.8 & \pm 11.1 & \pm 11.8 & \pm 10.5 & \pm 11.4 & \pm 10.4 \\
\% Male & 77.8 & 77.8 & 77.8 & 80.9 & 85.7 & 50.0 & 69.3 & 72.7 & 78.0 & 75.0 & 100 \\
AHI & 35.5 & 46.2 & 31.2 & 40.8 & 33.9 & 22.1 & 29.7 & 31.6 & 39.8 & 43.5 & 37.0 \\
(events/h) & \pm 12.5 & \pm 20.3 & \pm 10.5 & \pm 20.7 & \pm 16.7 & \pm 26.1 & \pm 23.7 & \pm 21.0 & \pm 23.3 & \pm 20.6 & \pm 25.5 \\
\hline \hline
\end{tabular}

of AHIs, with 79 patients having an AHI $<15$. Only the $\mathrm{SpO}_{2}$ and PPG signals of the PSG were used, these were recorded at $500 \mathrm{~Hz}$ for all datasets, but Dataset 1 contains older recordings which were downsampled for archiving. Therefore these signals were available at 200 or $50 \mathrm{~Hz}$. For further processing, all $\mathrm{SpO}_{2}$ signals were downsampled to $1 \mathrm{~Hz}$ and the PPG signals were resampled to $100 \mathrm{~Hz}$.

The presence of hypertension, hyperlipidemia, diabetes and atrial fibrillation was checked. In this study we will denote these conditions as "cardiovascular symptoms". The history of the patient was also inspected for the occurrence of "cardiovascular events" including heart failure, myocardial infarction and stroke. The patient group without any cardiovascular comorbidities (cardiac controls, C) and the group which experienced any cardiovascular event (E) will be used to select the most relevant cardiovascular markers. A classifier will be trained for this 2-class problem, using a subset of dataset 1 containing 45 cardiac control and 45 event patients. The behaviour of the intermediate group of patients suffering from any cardiovascular symptom (S) will also be discussed later.

\section{2. $\mathrm{SpO}_{2}$ feature extraction}

The $\mathrm{SpO}_{2}$ parameters were extracted as described in [5]. All oxygen desaturations were detected and 143 features were extracted from each oxygen desaturation. These features included simple time-domain (e.g. amplitude and length of desaturation), statistical (e.g. minimum and mean $\mathrm{SpO}_{2}$ value) and desaturation severity (e.g. area below $\mathrm{SpO}_{2}$ baseline) features computed on each desaturation. Moreover, quasi periodicity features (e.g. phase rectified signal averaging (PRSA) upslopes [6]) were computed on a 5 minute window around the desaturation. Afterwards, the feature values were averaged per patient for all desaturations with a minimal drop in $\mathrm{SpO}_{2}$ of $2 \%$.

\subsection{PPG feature extraction}

The PPG peaks were detected in the signal, and six PPG time series were extracted as described in [7]: the pulse rate, width and amplitude variability (PRV, PWV and PAV), the slope transit time (STT), the maximal pulse upslope and the area under the peak. The time-varying integral pulse frequency modulation (TVIPFM) model was used to estimate the PRV from the PPG beat midpoint occurrences in order to estimate the autonomic nervous system modulating signal [8]. Moreover, the instantaneous estimate of the power in the low frequency $(0.04-0.15 \mathrm{~Hz}$, LF) and high frequency (0.15-0.4 Hz, HF) bands of PRV were calculated using the point-process model [9].

In a 120 second time window around each oxygen desaturation, the median value, standard deviation and difference between minimum and maximum value (delta) were computed for each of these time series. Additionally, the degree of cardio-respiratory interactions was estimated by the power in the respiratory component of the PRV, obtained using orthogonal subspace projections [10], taking the high pass filtered PAV and PWV as surrogates of the respiratory signal. The obtained PPG parameters were then averaged per patient, as done for the $\mathrm{SpO}_{2}$ features.

\subsection{Feature selection and classification}

A total of 174 features were extracted from the pulse oximetry signals. In order to obtain an interpretable set containing the most meaningful features, a random forest based backwards wrapper was implemented. As performance measure, the difference in Cohen kappa values was used, when the samples of one feature are randomly permuted. In order to avoid highly correlated features, this value was multiplied by one minus the maximal inter-feature correlation as described in [11].

Using the selected features, a support vector machine (SVM) classifier with RBF kernel was trained in order to separate the patients without cardiovascular comorbidities from those who experienced a cardiovascular event. Since age is a known risk factor for cardiovascular disease, and the cardiovascular groups have an increased age compared to cardiac controls (as can be seen in Table 1), the classifier was trained with and without including the age as an extra parameter. 

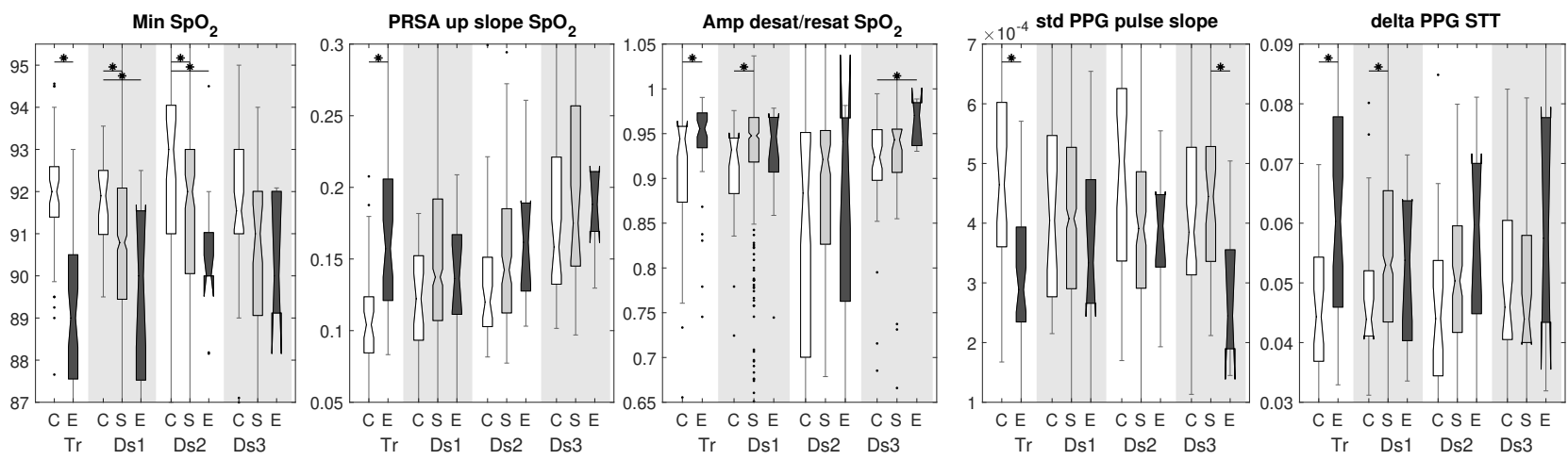

Figure 1. Overview of the selected features per dataset (From left to right: Train set (Tr), Dataset 1 (Ds1), Dataset 2 (Ds2) and Dataset 3 (Ds3)) and for each cardiovascular group (C, S, E). Statistical significant differences between groups $(\mathrm{p}<0.05)$ are marked with *.

\section{Results}

The proposed feature selection led to the selection of 3 $\mathrm{SpO}_{2}$ based features, including: the minimal $\mathrm{SpO}_{2}$ value, PRSA upslopes and ratio of amplitude of desaturation and resaturation. Additionally 2 PPG features were selected, the standard deviation of the pulse upslope and the difference in STT. Boxplots of these feature values per dataset, for the different cardiovascular categories (including the subjects with a cardiovascular symptom), can be found in Figure 1. The Spearman correlation coefficient between these features and the age is, respectively, $-0.25,-0.003$, $-0,17,-0,51$ and 0,16 .

Based on these features, different SVM classifiers were trained to assess the cardiovascular status of the patients (C vs E), using features of one or both modalities, and with or without including the age. The performance results on the different test sets can be found in Table 2, this table also includes the results of SVMs based on the AHI and/or age.

\section{Discussion}

The resulting feature set shows more severe oxygen desaturations in patients with cardiovascular events (lower min $\mathrm{SpO}_{2}$ ), a steeper upslope during the resaturation to baseline, and a larger amplitude of desaturation over the resaturation, which points to an incomplete recovery to baseline which might be followed by another $\mathrm{SpO}_{2}$ drop. The PPG features point to a decreased variability of the pulse upslope, this parameter is highly correlated with the delta PAV $\left(\mathrm{C}_{\text {Spearman }}=0.84\right)$. Since decreases in PAV have been linked to autonomic arousals [12], this could point to the fact that these patients have less arousals which could explain the more severe oxygen desaturations. The cardiac patients also have an increased difference in STT, this might be linked to the occurrence of irregular heartbeats.

As can be seen in Figure 1, the features are quite consis- tent over the different datasets and the cardiac symptoms (S) class lies in between the $\mathrm{C}$ and $\mathrm{E}$ class as expected.

When studying the classification results in Table 2, it can be concluded that the classifier based on $\mathrm{SpO}_{2}$ features including the age yields the best performance, an averaged area under the curve (AUC) of $77.7 \%$ is achieved. The PPG features do not give any added value to this classifier. The age is a known cardiovascular risk factor and the results show that age is a clear confounding parameter, a classification AUC of $70.6 \%$ can be achieved when taking only the age. However, adding the $\mathrm{SpO}_{2}$ features causes a large improvement in performance. These parameters only have a weak correlation with the age. The PPG features, on the other hand, do not give any added value to the age-based classifier. This might be explained by the stronger correlation between age and pulse upslope standard deviation.

When taking only the $\mathrm{AHI}$ as predictor, quasi random classification occurs with an AUC of $47.3 \%$. Adding the age to this classifier improves this towards $67.8 \%$, but this is still much lower than the performance achieved with the $\mathrm{SpO}_{2}$ based features. Only for the second dataset, acceptable results are obtained using the AHI and age, this is due to the fact that the cardiac control patients in this dataset tend to have low AHIs, often $<15$, see Table 1 .

In Figure 2, the posterior class probabilities of the $\mathrm{SpO}_{2}$ and age SVM are compared between the different datasets and cardiovascular groups. A clear differentiation between the cardiac control and event classes can be observed in all the datasets, the cardiovascular symptom groups are situated in between those two classes, as expected.

\section{Conclusion}

The results in this study suggest that $\mathrm{SpO}_{2}$ based features are an added value for the assessment of the cardiovascular status of sleep apnea patients. Patients with a cardiovascular comorbidity tend to have more severe oxygen 
Table 2. Cardiovascular comorbidity detection classification results between the cardiac control and events class.

\begin{tabular}{|l|ccc|ccc|ccc|ccc|}
\hline \hline & \multicolumn{3}{|c|}{ Train set } & \multicolumn{3}{|c|}{ Dataset 1 } & \multicolumn{3}{c|}{ Dataset 2 } & \multicolumn{3}{c|}{ Dataset 3 } \\
& Se & Sp & AUC & Se & Sp & AUC & Se & Sp & AUC & Se & Sp & AUC \\
\hline \hline $\mathrm{SpO}_{2}$ & 84.4 & 82.2 & 83.3 & 64.7 & 80.2 & 72.5 & 63.6 & 80.4 & 72.0 & 66.7 & 80.0 & 73.3 \\
$\mathrm{PPG}$ & 86.7 & 80.0 & 83.3 & 76.5 & 65.6 & 71.0 & 81.8 & 73.9 & 77.9 & 66.7 & 58.0 & 62.3 \\
$\mathrm{SpO}_{2}+\mathrm{PPG}$ & 82.2 & 82.2 & 82.2 & 64.7 & 78.1 & 71.4 & 63.6 & 78.3 & 70.9 & 66.7 & 78.0 & 72.3 \\
\hline $\mathrm{AHI}$ & 53.3 & 64.4 & 58.9 & 23.5 & 71.9 & 47.7 & 27.3 & 82.6 & 54.9 & 16.7 & 62.0 & 39.3 \\
$\mathrm{Age}$ & 88.9 & 71.1 & 80.0 & 64.7 & 76.0 & 70.4 & 63.6 & 82.6 & 73.1 & 66.7 & 70.0 & 68.3 \\
\hline $\mathrm{SpO}+$ Age & 86.7 & 84.4 & $\mathbf{8 5 . 6}$ & 70.6 & 85.4 & $\mathbf{7 8 . 0}$ & 72.7 & 89.1 & $\mathbf{8 0 . 9}$ & 66.7 & 82.0 & $\mathbf{7 4 . 3}$ \\
$\mathrm{PPG}_{2}$ Age & 86.7 & 75.6 & 81.1 & 64.7 & 76.0 & 70.4 & 63.6 & 82.6 & 73.1 & 66.7 & 70.0 & 68.3 \\
$\mathrm{SpO}_{2}+\mathrm{PPG}+$ Age & 84.4 & 84.4 & 84.4 & 70.6 & 83.3 & 77.0 & 72.7 & 84.8 & 78.8 & 66.7 & 82.0 & $\mathbf{7 4 . 3}$ \\
\hline $\mathrm{AHI}+$ Age & 82.2 & 80.0 & 81.1 & 52.9 & 85.4 & 69.2 & 63.6 & 91.3 & 77.5 & 33.3 & 80.0 & 56.7 \\
\hline \hline
\end{tabular}

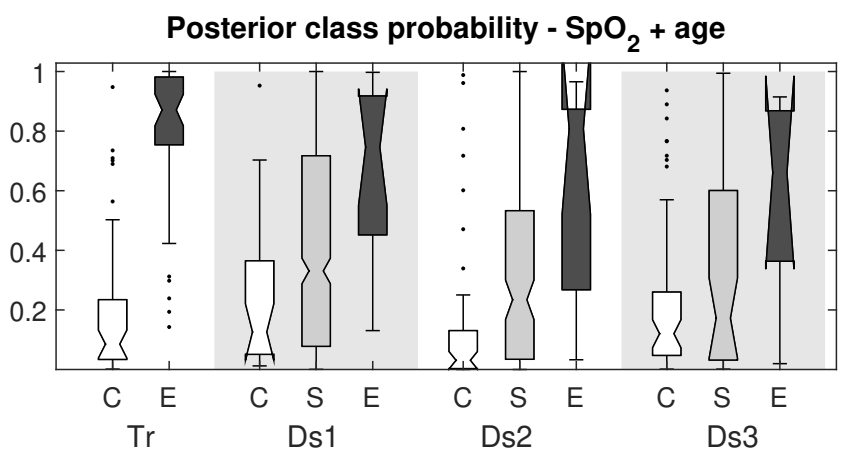

Figure 2. Posterior probability of the SVM classifier.

desaturations and often do not have a complete resaturation to their baseline $\mathrm{SpO}_{2}$ value. The investigated PPG based features did not show much added value to an age-based risk model.

\section{Acknowledgments}

This work was supported by: Agentschap Innoveren en Ondernemen (VLAIO) Project \#: SWT 150466 OSA+. imec funds 2017. imec ICON projects: ICON HBC.2016.0167. European Research Council: The research leading to these results has received funding from the European Research Council under the European Union's Seventh Framework Programme (FP7/2007-2013) / ERC Advanced Grant: BIOTENSORS ( ${ }^{\circ}$ 339804). This paper reflects only the authors' views and the Union is not liable for any use that may be made of the contained information. Carolina Varon is a postdoctoral fellow of the Research Foundation-Flanders (FWO).

\section{References}

[1] Berry RB, et al. Rules for scoring respiratory events in sleep: update of the 2007 aasm manual for the scoring of sleep and associated events. JCSM 2012;8(05):597-619.

[2] Marin JM, et al. Long-term cardiovascular outcomes in men with obstructive sleep apnoea-hypopnoea with or without treatment with continuous positive airway pressure: an observational study. The Lancet 2005;365(9464):1046-1053.

[3] Kendzerska T, et a. Obstructive sleep apnea and risk of cardiovascular events and all-cause mortality: a decadelong historical cohort study. PLoS medicine 2014; 11(2):e1001599.

[4] Heinzer R, et al. Prevalence of sleep-disordered breathing in the general population: the hypnolaus study. The Lancet Respiratory Medicine 2015;3(4):310-318.

[5] Deviaene $M$, et al. Automatic screening of sleep apnea patients based on the spo 2 signal. IEEE JBHI 2018; 23(2):607-617.

[6] Bauer A, et al. Phase-rectified signal averaging detects quasi-periodicities in non-stationary data. Physica A 2006; 364:423-434.

[7] Deviaene M, et a. Sleep apnea detection using pulse photoplethysmography. In CinC2018. IEEE, 2018; 1-4.

[8] Bailón R, et al. The integral pulse frequency modulation model with time-varying threshold: application to heart rate variability analysis during exercise stress testing. IEEE TBME 2010;58(3):642-652.

[9] Barbieri R, et al. A point-process model of human heartbeat intervals: new definitions of heart rate and heart rate variability. Am J Physiol Heart Circ Physiol 2005;288(1):H424H435.

[10] Varon C, et al. Unconstrained estimation of hrv indices after removing respiratory influences from heart rate. IEEE JBHI 2018;Early access; 10.1109/JBHI.2018.2884644.

[11] Deviaene $M$, et a. Feature selection algorithm based on random forest applied to sleep apnea detection. In EMBC2019. 2019; 1-4.

[12] Gil E, et al. Detection of decreases in the amplitude fluctuation of pulse photoplethysmography signal as indication of obstructive sleep apnea syndrome in children. BSPC 2008; 3(3):267-277.

Address for correspondence:

Margot Deviaene

ESAT-STADIUS, KU Leuven

Kasteelpark Arenberg 10, bus 2446, 3001 Leuven, Belgium

margot.deviaene@esat.kuleuven.be 